\title{
The Analysis of Digit Control Technique of Rocker and Crawler during Coalmine Transportation
}

\author{
Nianyuan Zhan \\ Guangxi Electrical Polytechnic Institute, Guilin, 530007, China
}

Keywords: Coalmine transmission. Digit control technology. Rocker. Crawler

\begin{abstract}
During the coalmine production, coalmine transmission is one of the most important tasks. There exists the efficiency problem between its rocker and crawler transmission to some extent. The traditional transmission can not meet the high effective requirements from the coalmine enterprises. Therefore, the numerical control technology has been introduced into the coal transmission. The numerical control technology can assure safety to some extent so as to improve its production efficiency. This essay is focused on the numerical control technology application in coalmine transmission and discussed its improvement in coalmine production.
\end{abstract}

\section{Introduction}

The main features of industrial production are procedural and workshop type, while the coalmine industry can meet the higher requirements - securitization. During the coalmine production, a lot of problems need to pay attention to. At the same time, a lot of technologies need to be brought into. The short-distance transportation of coalmine is the content that needs to be analyzed specially during the coalmine transportation. The distance is short, but a lot of technologies are involved. Therefore, the digit control technology has been brought into the crawler transportation of coalmine. The digit control technology is mainly applied in industrial production. Its technical advantages are oblivious, including improving the production efficiency of coalmine industry and production quality, so as to facilitate the industrial development to some extent. This paper explains the advantages of digit control technologies and discuss the application of it through the analysis of coalmine transportation.

\section{The transportation problem during coalmine production}

A lot of detailed work is involved in coalmine production. Because there are a lot of safety factors during the coalmine production, many problems have to be considered during the centralized coalmine production. In many production tasks, many projects need to be supervised specially by coalmine transportation department. As a result, the transportation task for coalmine also needs more techniques. During the coalmine transportation, how to conduct efficient transportation? What involved technology fields are worth exploring?

\section{Analysis of coalmine transportation task}

General speaking, there are two kinds of coalmine transportation tasks involved in coalmine production. The first one is long distance transportation. After the coalmine which will be short-term stored is produced, it will be transported to processing workshop by transport vehicles to reprocess. This kind of transportation task is relatively simple, involving simple freight transportation. The second one is production and transportation. The production work in the coalmine production lies in mines. Because most of mines in China are inclined mines, the produced coalmine has to be transported to mines above, so as to provide enough space for reproduction and guarantee the working environment like production line. Therefore, during the second coalmine transportation, the transportation distance is short, but needs more requirements, including some innovative and high technologies. For this reason, the focus is to discuss the crawler transportation during the coalmine transportation tasks. The crawler transportation refers to transport the packed coalmine to mines above through crawler-type conveyor and rocker \& crawler attachment to reprocess. 


\section{The rocker and crawler transportation for coalmine}

According to the above analysis, it is not difficult to find: although the long-distance transportation of coalmine is very large, the transportation task is simple with low technological content. However, as for the short-distance transportation task, it uses rocker and crawler transportation mode. As for the rocker and crawler transportation mode, the transportation distance is short, but it involves a lot of technological theories. The dip angle of rocker has to be analyzed. During the coalmine production, the dip angle needs to be discussed to obtain a fixed value. If the coalmine production is conducted with budget program, the rocker's position shall be designed and confirmed according to the progress of production. Moreover, the requirements on crawler and the estimation of friction coefficient shall avoid the sideslipping or falling off of coalmines on the crawler. Consequently, the crawler and rocker for coalmine is short-distance transportation, but it involves a lot of technologies. The coalmine designer has to conduct detail calculation, so as to guarantee the safety and smoothness of transportation.

\section{The analysis of digit control technique}

With the continuous development of technologies, a lot of advanced industrial technologies have been brought into industrial production. The digit control technique is one of these technologies. The digit control technique does extremely well in many fields. The focus of the digit control technique is control. With the continuous improvement of technologies, the intelligentialized digit control techniques begin to emerge, which are applied to industrial production gradually, so as to modernize the coalmine production projects. What is the application of digit control techniques during the coalmine production? What is the application of technology core during the rocker and crawler transportation of coalmine?

\section{The analysis of concept for digit control technique}

Before analyzing the application of digit control technique into coalmine transportation, we shall know the concept of digit control technique. The digit control technique, which is called digital control by people, is a kind of technology that uses the digital control to conduct automatic control. Especially for industrial production, besides the manufacturing shop needs manpower, there are a lot of safety problems in the production, causing big error and labor waste to some extent. During the industrial process, we have a high requirement on precision. Therefore, the deviation is common in manufacturing shop. However, if the small errors are pursued far too much, it will cause the corresponding effect on the production efficiency. As a result, the digital control technology, which will encode the executive program and control program, realizes the process of automatic control technology.

The analysis of the introduction of digital control technology into coalmine production

During the traditional industrial processes, a lot of manpower and material resources are required. For the industrial enterprise, there are two drawbacks at the aspect of using manpower.

First all of, the manpower in large use will increase the staff cost, causing the increase of cost. Moreover, there are a lot of disadvantages in staff production. For example, the decrease of production quality is caused by various elements, such as staff's autonomy and responsibility.

Secondary, for the error of products production, the manpower in large use can not be controlled strictly. The staff's industrial production is bound to have error. This error is the unavoidable staff error. However, high precision is required by industrial production. The product quality is affected to some extent. Moreover, the staff needs rest. Although the work rotations is suitable, it will increase the pressure of staff and increase the production cost to some extent, which is not applicable for the long-term development of industrial enterprise.

\section{The analysis of transportation task for coalmine production}

The coalmine production is completely different from workshop production. The transportation task for coalmine production shall be conducted through crawler and rocker. Although it can solve the staff's handling, the traditional rocker transportation can not achieve the clear transportation. Rocker is the lever of transportation, and the crawler is the main support point of transportation. Both 
of them need a power source to provide power. However, there will be a problem in the power supply process. Therefore, there are some drawbacks in traditional coalmine crawler transportation, which can not meet the requirements for high efficient transport.

\section{The analysis of the reason for introduction the digit control technique into coalmine transportation}

Why do introduce the digit control technique into coalmine transportation? From the point of traditional analysis angle, the short-distance transportation of coalmine is relatively simple; therefore, it is not necessary to conduct a large scale of technology input. However, with the improvement of technology and the continuous development of digital control technology, the application of digit control technique into coalmine transportation is promoted. As for the traditional technology, because there is not control system, the running speed of the crawler will be unstable during the transpiration, causing the sliding down of coalmine. As a result, it will need the staff's transpiration. Therefore, this kind of phenomena can be decreased by digit control technique to a large extent, increasing the transportation efficiency of coalmine. As a result, the whole operation process, the introduction of digit control technique can resolve the operation problem of production line. If the ongoing operation can be guaranteed, then the whole production efficiency can be improved to a large extent, which is meaningful for coalmine production engineering.

\section{Analysis of the Application of Digit Control Technology in Coalmine Transportation}

With the introducing of digit control technology, its control of rocker and crawler transportation is mainly expressed as follows:

Firstly, it can help the angle control of rocker transportation. The digit control technology applied in coalmine transportation can set its parameters before so as to keep its intelligent automatic control on rocker's angle. Traditional rocker is kept in a state of fixed angle. Therefore, it is necessary to readjust angle while the angle of the coalmine is changing. The digit control technology is so flexible that it can suit the telescopic rocker. When the angle of coalmine is changing, it can adjust the angle of rocker so as to assure the normal carriage of coalmine.

Secondly, it can help keep the power control. The most important guarantee during the crawler belt transportation of coalmine is the constant power assurance which is the promise of its constant transportation speed. The constant transportation speed can keep its safety, smooth and steady which enables its high efficiency. During the traditional transportation, the voltage instability has caused the pause and stop of transmitting crawler. The digit control technology can have a solid control of the voltage with constant voltage procedure which connected to the voltage regulator, in order to keep constant control of voltage, which leads a steady output of voltage. What's more, when the quality of work as large as overload transportation, the lack of automatic cutting system will cause damage to the power source, with the circuit damaging. By the introduction of digit control technology, the nominal voltage can be set and protect the voltage from the overworking number. Once it works over its nominal voltage, digit control program will cut off power automatically and slow the transmitting rocker down to quiet, which protects coalmine from creeping down.

All in all, the introduction of numerical technology during the coalmine transportation has to the great extent realized the mobility of coal production, improved the producing efficiency and provided very strong technique support within the whole coalmine producing procedure.

\section{Conclusion}

The digit control technology is a kind of technology that operates the equipment to conduct automatic control by digitization procedure entering. It is commonly used in industrial fields. During the coalmine production, the digit control technology can also be used in many fields. But for the crawler transportation for coalmine, it is more important to use digit control technology. The speed of the crawler transportation shall be in the uniform state, so as to guarantee the normal transportation of coalmine. The smoothness of coalmine transportation can be achieved through digit control technique which will control the power by adjusting the angle of rocker in time. The in-line operation of coalmine production can be guaranteed to a large extent. As for the digit control technique, it shall 
be applied to the coalmine production, so as to guarantee the normal production of coalmine, which is necessary. As a result, the introduction of digit control technique improves the production efficiency of coalmine and guarantees the efficient production of coalmine.

\section{References}

[1] Quanhai Zhao and Jing Hu: Building Operation System to Prevent Production Risks in Coalmine by the Market Mechanism, Journal of Shanxi Radio \& TV University, 2013(01):71-73

[2] Bo Gao and Naiping Niu: Enterprise Network Construction and Information Safety for Coalmine Production, Electron World, 2013 (01):89-90

[3] Qingyu Wang: The Room for Video Monitoring Technique Applied in Coalmine Production Safety, Communications World, 2012 (47):35-35

[4] Jianguo Shen: Research on Coalmine Security Diagnosis System Based on Fuzzy Logic Inference, Coal Engineering, 2012(12):56-57

[5] Shumao Pei: The Application of Mapping Information System for Coalmine Production, Inner Mongolia Coal Economy, 2012(12):112-114 